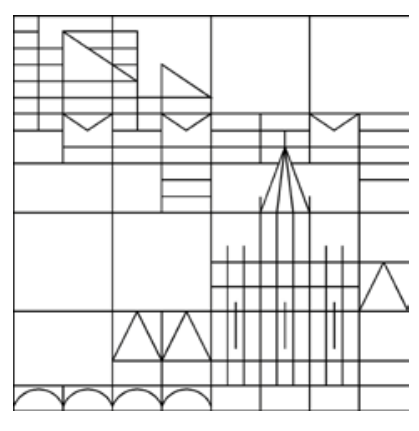

\title{
Global existence and decay property of the Timoshenko system in thermoelasticity with second sound
}

\author{
Reinhard Racke \\ Belkacem Said-Houari
}

Konstanzer Schriften in Mathematik

Nr. 295, Februar 2012

ISSN 1430-3558 



\title{
Global existence and decay property of the Timoshenko system in thermoelasticity with second sound
}

\author{
Reinhard RACKe *\& BelKaCEM SAID-Houari ${ }^{\dagger}$
}

\begin{abstract}
Our main focus in the present paper is to study the asymptotic behavior of a nonlinear version of the Timoshenko system in thermoelasticity with second sound. As it has been already proved in [38], the linear version of this system is of regularity-loss type. It is well known ([10], [13], [19]) that the regularity-loss property of the linear problem creates difficulties when dealing with the nonlinear problem. In fact, the dissipative property of the problem becomes very weak in the high frequency region and as a result the classical energy method fails. To overcome this difficulty and following [13] and [14], we use an energy method with negative weights to create an artificial damping which allows us to control the nonlinearity. We prove that for $0 \leq$ $k \leq[s / 2]-2$ with $s \geq 8$, the solution of our problem is global in time and decays as $\left\|\partial_{x}^{k} U(t)\right\|_{2} \leq C(1+t)^{-1 / 4-k / 2}$, provided that the initial datum $U_{0} \in H^{s}(\mathbb{R}) \cap L^{1}(\mathbb{R})$.
\end{abstract}

Keywords: Timoshenko systems; thermoelasticity; second sound; decay rate; regularityloss.

AMS Classification: 35B37, 35L55, 74D05, 93D15, 93D20.

\section{Introduction}

In this paper, we consider the dissipative Timoshenko system in thermoelasticity of second sound

$$
\left\{\begin{array}{l}
\varphi_{t t}-\left(\varphi_{x}-\psi\right)_{x}=0 \\
\psi_{t t}-\sigma\left(\psi_{x}\right)_{x}-\left(\varphi_{x}-\psi\right)+\beta \theta_{x}+\alpha \psi_{t}=0 \\
\theta_{t}+\kappa q_{x}+\beta \psi_{t x}=0 \\
\tau_{0} q_{t}+\delta q+\kappa \theta_{x}=0
\end{array}\right.
$$

${ }^{*}$ Department of Mathematics and Statistics, University of Konstanz, 78457 Konstanz, Germany. E-mail: reinhard.racke@uni-konstanz.de.

${ }^{\dagger}$ Division of Mathematical and Computer Sciences and Engineering, King Abdullah University of Science and Technology (KAUST), Thuwal, Saudi Arabia. E-mail: belkacem.saidhouari@kaust.edu.sa 
where $t \in(0, \infty)$ denotes the time variable and $x \in \mathbb{R}$ is the space variable, the functions $\varphi$ and $\psi$ are the displacement of the solid elastic material, the function $\theta$ is the temperature difference, $q=q(t, x) \in \mathbb{R}$ is the heat flux, and $\gamma, \tau_{0}, \delta, \kappa$ and $\beta$ are positive constants, and $\sigma$ is a smooth function such that $\sigma^{\prime}(\eta)>0$, for any $\eta>0$, with

$$
\sigma^{\prime}(0)=a^{2}
$$

defining $a>0$. We consider the following initial conditions

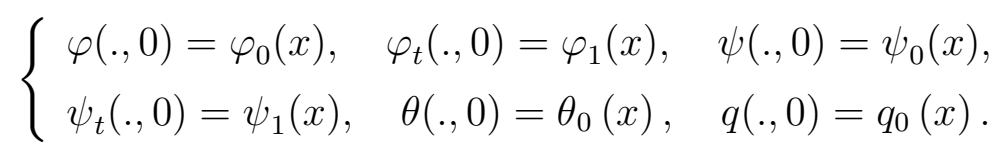

Before going on, let us recall some other works related to the problem we address. In the classical theory of thermoelasticity, the behavior of an elastic heat body can be described by a coupled system of hyperbolic-parabolic type, where the classical Fourier model of heat conduction is used. This law assumes the flux $q$ to be proportional to the gradient of the temperature $\theta$ at the same time $t$,

$$
q+\kappa \nabla \theta=0
$$

where $\kappa>0$ is the thermal conductivity depends on the properties of the material. This hyperbolic-parabolic system is interesting due to its large applications in mechanics, physics and engineering problems.

Modeling heat conduction with the so-called Fourier law, which assumes the flux $q$ to be proportional to the gradient of the temperature $\nabla \theta$ at the same time $t$ as in (1.3), leads to the paradox of infinite heat propagation speed. That is, any local thermal disturbance can have an instantaneous effect everywhere in the medium. In other words, it is clear that equation (1.3) together with the energy equation of the heat conduction

$$
\rho_{3} \theta_{t}+\varrho \operatorname{div} q=0
$$

yields the classical heat transport equation (of parabolic type)

$$
\rho_{3} \theta_{t}-\kappa \varrho \Delta \theta=0
$$

allows an infinite speed for thermal signals. To overcome this drawback, a number of modifications of the basic assumption on the relation between the heat flux and the temperature have been made, such as: Cattaneo law, Gurtin and Pipkin theory, Jeffreys law, Green and Naghdi theory and others. The common feature of these theories is that all lead to hyperbolic differential equation and permit transmission of heat flow as thermal waves at finite speed, see $[5,18]$ for more details.

Here, we consider the Cattaneo law,

$$
\tau_{0} q_{t}+q+\kappa \nabla \theta=0, \quad\left(\tau_{0}>0, \text { relatively small }\right)^{1},
$$

\footnotetext{
${ }^{1}$ The constant $\tau_{0}$ represents the time lag needed to establish the steady state of the heat conduction in an element of volume when a temperator gradient is suddenly imposed on that element. See the survey paper [4] for more details. Moreover, and as it was shown in [6], in real solid materials the constant $\tau_{0}$ is likely to be extremely small compared with time lengths.
} 
replacing the Fourier law (1.3).

Equation (1.6) was proposed by Cattaneo in [3] in order to correct the paradox of instantaneous propagation of thermal disturbances predicted by Fourier's theory of heat conduction. Also, formula (1.6) is the most obvious and simplest generalization of Fourier's law that gives rise to finite speeds of propagation. Indeed, from (1.4) and (1.6), we get the telegraph equation

$$
\rho_{3} \theta_{t t}-\frac{\varrho \kappa}{\tau_{0}} \Delta \theta+\frac{\rho_{3}}{\tau_{0}} \theta_{t}=0
$$

which is an equation of hyperbolic type and predicts a finite speed equals to $\left(\varrho \kappa /\left(\rho_{3} \tau_{0}\right)\right)^{1 / 2}$ for the heat propagation.

The classical thermoelastic systems has been investigated by many authors (cf. the book [17]). The system of equations describing the coupling of elastic and thermal behavior of a body, where the heat flux obeys Cattaneo's law, is given (in the linear one dimensional case) by

$$
\left\{\begin{array}{c}
u_{t t}-b u_{x x}+\gamma \theta_{x}=0 \\
\theta_{t}+\kappa q_{x}+\gamma u_{t x}=0 \\
\tau_{0} q_{t}+\delta q+\kappa \theta_{x}=0
\end{array}\right.
$$

where $b, \gamma, \kappa, \delta$ and $\tau_{0}$ are positive constants.

Problems related to (1.8) have been studied in recent years, see [25, 32, 33, 34, 36, 41]. In most of these papers, it has been proved that the behavior of problem (1.8) is similar to the behavior of the classical ${ }^{2}$ thermoelastic system (i.e. system (1.8) with $\tau_{0}=0$ ), cf. the survey [34].

The Timoshenko system with heat conduction of Cattaneo's law, problem (1.1), is particularly interesting since the behavior of the Timoshenko system in thermoelasticity of second sound is different from the one in classical thermoelasticity. In fact the first example in this direction has been given in [8]. There, Fernández Sare and Racke investigated the system

$$
\left\{\begin{array}{l}
\rho_{1} \varphi_{t t}-k\left(\varphi_{x}+\psi\right)_{x}=0 \\
\rho_{2} \psi_{t t}-b \psi_{x x}+k\left(\varphi_{x}+\psi\right)+\beta \theta_{x}=0 \\
\rho_{3} \theta_{t}+\gamma q_{x}+\delta \psi_{t x}=0 \\
\tau_{0} q_{t}+q+\kappa \theta_{x}=0
\end{array}\right.
$$

where $(x, t) \in(0, L) \times(0, \infty)$ and $\varphi=\varphi(t, x)$ is the displacement vector, $\psi=\psi(t, x)$ is the rotation angle of the filament, $\theta=\theta(t, x)$ is the temperature difference, $q=q(t, x)$ is the heat flux vector, $\rho_{1}, \rho_{2}, \rho_{3}, b, k, \gamma, \delta, \kappa, \mu, \tau_{0}$ are positive constants. They showed that this system is no longer exponentially stable even if the wave speeds of the first two equations in

\footnotetext{
${ }^{2}$ By classical we mean the Fourier law of heat conduction.
} 
(1.9) are equal, that is

$$
\frac{k}{\rho_{1}}=\frac{b}{\rho_{2}} .^{3}
$$

In contrast, the Timoshenko system with Fourier's law (i.e. $\tau_{0}=0$ in (1.9)) is exponentially stable provided that (1.10) is fulfilled. This later result has been proved by Muñoz Rivera and Racke in [27].

An even more surprising result is that the Cattaneo coupling even "destroys" exponential stability. More precisely, Muñoz Rivera and Fernández Sare [37] and Messaoudi and SaidHouari [23], considered a Timoshenko type system with past history acting only in one equation. They looked into the following problem

$$
\left\{\begin{array}{l}
\rho_{1} \varphi_{t t}-k\left(\varphi_{x}+\psi\right)_{x}=0 \\
\rho_{2} \psi_{t t}-b \psi_{x x}+\int_{0}^{\infty} g(t) \psi_{x x}(t-s, .) d s+k\left(\varphi_{x}+\psi\right)=0
\end{array}\right.
$$

together with homogenous boundary conditions in a bounded domain, and showed that the dissipation given by the history term is strong enough to stabilize the system exponentially if and only if (1.10) holds and $g$ decays exponentially. Also a polynomial decay result has been shown in [23] for $g$ decaying polynomially. It has been proved the exponential stability of the system in [37], while the Cattaneo law "destroys" this property, as it has been shown in [8]. For this reason some additional damping terms might be necessary to restore the exponential stability of system (1.9). This situation has been studied by Messaoudi et al. [22], where a nonlinear version of (1.9) has been also considered and a damping term of the form $\mu \varphi_{t}$ has been introduced. Namely, they looked at the following problem

$$
\left\{\begin{array}{l}
\rho_{1} \varphi_{t t}-\sigma\left(\varphi_{x}, \psi\right)_{x}+\mu \varphi_{t}=0 \\
\rho_{2} \psi_{t t}-b \psi_{x x}+k\left(\varphi_{x}+\psi\right)+\beta \theta_{x}=0 \\
\rho_{3} \theta_{t}+\gamma q_{x}+\delta \psi_{t x}=0 \\
\tau_{0} q_{t}+q+\kappa \theta_{x}=0
\end{array}\right.
$$

where $(x, t) \in(0, L) \times(0, \infty), \mu>0$, and the nonlinear function $\sigma$ is assumed to be sufficiently smooth and satisfies

$$
\sigma_{\varphi_{x}}(0,0)=\sigma_{\psi}(0,0)=k
$$

and

$$
\sigma_{\varphi_{x} \varphi_{x}}(0,0)=\sigma_{\varphi_{x} \psi}(0,0)=\sigma_{\psi \psi}=0 .
$$

Several exponential decay results for both linear and nonlinear cases have been established without the assumption (1.10).

\footnotetext{
${ }^{3}$ This condition is significant only from the mathematical point of view since in practice the velocities of waves propagations are always different.
} 
According to what we have explained before, the presence of the linear damping term $\alpha \psi_{t}$ in (1.1) is justified and seems necessary to preserve the decay rate of the pure Timoshenko system.

For the pure Timoshenko system (i.e. without heat conduction) in bounded domain, there is an extensive literature. The interested reader is referred to $[1,21,24,28,29,40]$ for the Timoshenko systems with frictional damping and to [2, 9, 26, 37] for Timoshenko systems with viscoelastic damping.

It is well known that the proof of the stability results of the Timoshenko systems in a bounded domain is somehow based on the Poincaré inequality and the type of the boundary conditions. But in the whole space $\mathbb{R}$ there are only few results, to our knowledge.

In [12], Ide, Haramoto and Kawashima investigated the problem

$$
\begin{cases}\varphi_{t t}(t, x)-\left(\varphi_{x}-\psi\right)_{x}(t, x)=0, & (t, x) \in \mathbb{R}^{+} \times \mathbb{R}, \\ \psi_{t t}(t, x)-a^{2} \psi_{x x}(t, x)-\left(\varphi_{x}-\psi\right)(t, x)+\mu \psi_{t}(t, x)=0, & (t, x) \in \mathbb{R}^{+} \times \mathbb{R}, \\ \left(\varphi, \varphi_{t}, \psi, \psi_{t}\right)(0, x)=\left(\varphi_{0}, \varphi_{1}, \psi_{0}, \psi_{1}\right), & x \in \mathbb{R},\end{cases}
$$

where $t$ denotes the time variable and $x$ is the space variable, the function $\varphi$ and $\psi$ are the displacement and the rotation angle of the beam respectively, $a$ and $\mu$ are positive constants. They used Fourier analysis to obtain precise decay rates for spacial $L^{2}$-norm of solutions to the linear problem (1.13). More precisely, they proved that if $a=1$, then the solution of (1.13) decays like:

$$
\left\|\partial_{x}^{k} U(t)\right\|_{2} \leq C(1+t)^{-1 / 4-k / 2}\left\|U_{0}\right\|_{1}+C e^{-c t}\left\|\partial_{x}^{k} U_{0}\right\|_{2}
$$

where $U=\left(\varphi_{x}-\psi, \varphi_{t}, a \psi_{x}, \psi_{t}\right)^{T}$. While if $a \neq 1$, then system (1.13) is of regularity-loss type and the solutions decay as:

$$
\left\|\partial_{x}^{k} U(t)\right\|_{2} \leq C(1+t)^{-1 / 4-k / 2}\left\|U_{0}\right\|_{1}+C(1+t)^{-l / 2}\left\|\partial_{x}^{k+l} U_{0}\right\|_{2},
$$

where the parameters $k$ and $l$ in (1.14) and (1.15) are non-negative integers, and $C$ and $c$ are positive constants.

Estimate (1.15) indicates that system (1.13) is of regularity-loss type, which means that the decay rate $(1+t)^{-1 / 4-k / 2}$ can be obtained only under the additional assumption that $l>1 / 2+k$ more derivates of $U_{0}$ exist.

The work in [12] was followed by [13] where Ide and Kawashima generalized the above decay results to a nonlinear version, where they considered $\sigma\left(\psi_{x}\right)_{x}$ instead of $a^{2} \psi_{x x}$ in (1.13) where $\sigma(\eta)$ is a smooth function of $\eta$ such that $\sigma^{\prime}(\eta)>0$. In fact, they combined weighted energy estimates with the result of [12] to establish global existence for the nonlinear problem. They also obtained precise decay rates for the solutions, under the smallness condition on the initial data in $H^{s} \cap L^{1}$ with suitably large $s$, (in fact for $s \geq 6$ ).

In [35], we analyzed system (1.13) with a nonlinear term of the form $|\psi|^{p},(p>1)$ acting on the right hand side of the second equation. We extended the decay results obtained in [12] 
so that, for initial data $U_{0} \in H^{s}(\mathbb{R}) \cap L^{1, \gamma}(\mathbb{R})$ with a suitably large $s$ and $\gamma \in[0,1]$, solutions decay faster than those given in [12]. In addition, we proved a global existence result and some decay estimates of the semilinear system for $p>12$. Recently, Said-Houari and Kasimov [38] proved that, for the Cauchy problem, the coupling of the linear Timoshenko system with the Fourier law preserves the decay properties (1.14) and (1.15). While for the Cattaneo law, they proved that the regularity-loss type estimate (1.15) appears independently on the wave speed $a$.

It is the purpose of this paper to extend the result in [13] to the initial value problem (1.1)-(1.2). Since, the dissipative property of the problem becomes very weak in the high frequency region and as result the classical energy method fails. To overcome this difficulty and following [13] and [14], we use an energy method with negative weights to create an artificial damping which allows us to control the nonlinearity. Here, a substantial modification of the energy functionals resp. the Lyapunov functional will be necessary. We prove that for $0 \leq k \leq[s / 2]-2$ with $s \geq 8$, the solution $U=\left(\varphi_{x}-\psi, \varphi_{t}, a \psi_{x}, \psi_{t}, \theta, \tau_{0} q\right)^{T}$ of our problem is global in time and decays as $\left\|\partial_{x}^{k} U(t)\right\|_{2} \leq C(1+t)^{-1 / 4-k / 2}$, provided that the initial datum $U_{0} \in H^{s}(\mathbb{R}) \cap L^{1}(\mathbb{R})$.

This paper is organized as follows: In section 2, we fix notations and for the convenience of the reader, we recall without proofs some useful technical Lemmas. In section 3, we state our main result, while section 4 is devoted to the proof of our main result.

\section{Preliminaries}

In this section, we introduce some notations and some technical lemmas to be used throughout this paper. Throughout this paper, $\|\cdot\|_{q}$ and $\|\cdot\|_{H^{l}}$ stand for the $L^{q}(\mathbb{R})$-norm $(1 \leq q \leq \infty)$ and the $H^{l}(\mathbb{R})$-norm. Also, for $\gamma \in[0, \infty)$, we define the weighted function space $L^{p, \gamma}(\mathbb{R})$ as follows: $u \in L^{p, \gamma}(\mathbb{R})$ iff $u \in L^{p}(\mathbb{R})$ and

$$
\|u\|_{p, \gamma}^{p}=\int_{\mathbb{R}}(1+|x|)^{\gamma}|u(x)|^{p} d x<\infty
$$

Let us also denote by $\hat{f}=\mathcal{F}(f)$ the Fourier transform of $f$ with inverse $\mathcal{F}^{-1}$ :

$$
\hat{f}(\xi)=\mathcal{F}(f)(\xi)=\int_{\mathbb{R}} f(x) e^{-i \xi x} d x
$$

Next, we introduce the following lemma, which can be found, for example in [20,39], cp. also Lemma 7.4 in [31].

Lemma 2.1 Let $a>0$ and $b>0$ be constants. If $\max (a, b)>1$, then

$$
\int_{0}^{t}(1+t-s)^{-a}(1+s)^{-b} d s \leq C(1+t)^{-\min (a, b)}
$$


If $\max (a, b)=1$, then

$$
\int_{0}^{t}(1+t-s)^{-a}(1+s)^{-b} d s \leq C(1+t)^{-\min (a, b)} \ln (2+t) .
$$

If $\max (a, b)<1$, then

$$
\int_{0}^{t}(1+t-s)^{-a}(1+s)^{-b} d s \leq C(1+t)^{1-a-b}
$$

Furthermore, the next Lemma has been proved for instance in [10, Lemma 4.1].

Lemma 2.2 Let $1 \leq p, q, r \leq \infty$ and $1 / p=1 / q+1 / r$. Then, we have

$$
\left\|\partial_{x}^{k}(u v)\right\|_{p} \leq C\left(\|u\|_{p}\left\|\partial_{x}^{k} v\right\|_{r}+\|v\|_{q}\left\|\partial_{x}^{k} u\right\|_{r}\right), \quad k \geq 0
$$

and

$$
\left\|\left[\partial_{x}^{k}, u\right] v_{x}\right\|_{p} \leq C\left(\left\|u_{x}\right\|_{p}\left\|\partial_{x}^{k} v\right\|_{r}+\left\|v_{x}\right\|_{q}\left\|\partial_{x}^{k} u\right\|_{r}\right), \quad k \geq 1
$$

\section{Main results}

In this section, we present the results on the global existence and the asymptotic decay of the solutions of problem (1.1)-(1.2). To this end, we write our system (1.1)-(1.2) as a first-order system. Indeed, we introduce the following variables:

$$
v:=\varphi_{x}-\psi, \quad u:=\varphi_{t}, \quad z:=a \psi_{x}, \quad y:=\psi_{t}, \quad w:=\tau_{0} q,
$$

where we recall that $a^{2}=\sigma^{\prime}(0)$. Consequently, system (1.1) can be rewritten as the following first-order hyperbolic system:

$$
\left\{\begin{array}{l}
v_{t}-u_{x}+y=0 \\
u_{t}-v_{x}=0 \\
z_{t}-a y_{x}=0 \\
y_{t}-\sigma(z / a)_{x}-v+\alpha y+\beta \theta_{x}=0 \\
\theta_{t}+\frac{\kappa}{\tau_{0}} w_{x}+\beta y_{x}=0 \\
w_{t}+\frac{\delta}{\tau_{0}} w+\kappa \theta_{x}=0
\end{array}\right.
$$

and the initial conditions (1.2) take the form

$$
(v, u, z, y, \theta, w)(x, 0)=\left(v_{0}, u_{0}, z_{0}, y_{0}, \theta_{0}, w_{0}\right)
$$


where

$$
v_{0}=\varphi_{0, x}-\psi_{0}, \quad u_{0}=\psi_{1}, \quad z_{0}=a \psi_{0, x}, \quad y_{0}=\psi_{1}, \quad w_{0}=\tau_{0} q_{0} .
$$

System (3.1)-(3.2) is equivalent to the first-order system

$$
\left\{\begin{array}{l}
U_{t}+F(U)_{x}+L U=0 \\
U(x, 0)=U_{0}
\end{array}\right.
$$

where $U:=(v, u, z, y, \theta, w)^{T}, F(U):=-\left(u, v, a y, \sigma(z / a)-\beta \theta,-\frac{\kappa}{\tau_{0}} w-\beta y,-\kappa \theta\right)^{T}$ and $L$ is defined as

$$
L:=\left(\begin{array}{cccccc}
0 & 0 & 0 & 1 & 0 & 0 \\
0 & 0 & 0 & 0 & 0 & 0 \\
0 & 0 & 0 & 0 & 0 & 0 \\
-1 & 0 & 0 & \alpha & 0 & 0 \\
0 & 0 & 0 & 0 & 0 & 0 \\
0 & 0 & 0 & 0 & 0 & \frac{\delta}{\tau_{0}}
\end{array}\right)
$$

and $U_{0}:=\left(v_{0}, u_{0}, z_{0}, y_{0}, \theta_{0}, w_{0}\right)^{T}$. It is clear that $U^{T} L U=\alpha y^{2}+\frac{\delta}{\tau_{0}} w^{2} \geq 0$. Thus, $L$ is a non-negative definite but it is not real symmetric.

The linearized problem of (3.3) can be obtained by taking the Jacobian of $F$ in $U=0$. Thus, we get the problem

$$
\left\{\begin{array}{l}
U_{t}+A U_{x}+L U=0 \\
U(x, 0)=U_{0}
\end{array}\right.
$$

where the matrix $A$ is defined as

$$
A:=D_{U} F(0)=-\left(\begin{array}{cccccc}
0 & 1 & 0 & 0 & 0 & 0 \\
1 & 0 & 0 & 0 & 0 & 0 \\
0 & 0 & 0 & a & 0 & 0 \\
0 & 0 & a & 0 & -\beta & 0 \\
0 & 0 & 0 & -\beta & 0 & \frac{\kappa}{\tau_{0}} \\
0 & 0 & 0 & 0 & \kappa & 0
\end{array}\right) .
$$

The six real eigenvalues of $A$ are

$$
\lambda_{1|2| 3 \mid 4}= \pm \frac{1}{2} \sqrt{\frac{2}{\tau_{0}}} \sqrt{\eta_{1} \pm \sqrt{\eta_{2}}}, \quad \lambda_{5 \mid 6}= \pm 1,
$$

where

$$
\eta_{1}=a^{2} \tau_{0}+\kappa^{2}+\beta^{2} \tau_{0}, \quad \eta_{2}=\left(a^{2} \tau_{0}-\kappa^{2}\right)^{2}+2 a^{2} \beta^{2} \tau_{0}^{2}+2 \kappa^{2} \beta^{2} \tau_{0}+\beta^{4} \tau_{0}^{2} .
$$

Consequently, system (3.4) is a hyperbolic system in the main part $U_{t}+A U_{x}=0$ with a damping term $L U$. This late system has been investigated in [38], where the following result has been proved. 
Theorem 3.1 ([38]) Let $s$ be a nonnegative integer and assume that $U_{0} \in H^{s}(\mathbb{R}) \cap L^{1}(\mathbb{R})$. Then the solution $U$ of problem (3.4) satisfies the following decay estimates:

$$
\left\|\partial_{x}^{k} U(t)\right\|_{2} \leq C(1+t)^{-1 / 4-k / 2}\left\|U_{0}\right\|_{L^{1}}+C(1+t)^{-l / 2}\left\|\partial_{x}^{k+l} U_{0}\right\|_{2}
$$

Moreover, if $U_{0} \in H^{s}(\mathbb{R}) \cap L^{1, \gamma}(\mathbb{R})$, with $\gamma \in[0,1]$, then the solution $U$ of problem (3.4) satisfies the following decay estimates:

$$
\begin{aligned}
\left\|\partial_{x}^{k} U(t)\right\|_{2} \leq & C(1+t)^{-1 / 4-k / 2-\gamma / 2}\left\|U_{0}\right\|_{L^{1, \gamma}}+C(1+t)^{-l / 2}\left\|\partial_{x}^{k+l} U_{0}\right\|_{2} \\
& +C(1+t)^{-1 / 4-k / 2}\left|\int_{\mathbb{R}} U_{0}(x) d x\right|,
\end{aligned}
$$

where $k$ and $l$ are non-negative integers satisfying $k+l \leq s$ and $C$ and $c$ are two positive constants.

The estimate in (3.6) becomes optimal under the condition $\int_{\mathbb{R}} U_{0}(x) d x=0$.

Remark 3.2 For $\gamma \in \mathbb{N}$ and $U_{0} \in H^{s}(\mathbb{R}) \cap L^{1,2(\gamma+1)}(\mathbb{R})$ satisfying

$$
\int_{\mathbb{R}} x^{m} U_{0}(x) d x=0, \quad m=0, \ldots, 2 \gamma,
$$

one has the following decay estimate with stronger decay,

$$
\begin{aligned}
\left\|\partial_{x}^{k} U(t)\right\|_{2} \leq & C(1+t)^{-1 / 4-k / 2-(2 \gamma+1) / 2}\left(\left\|U_{0}\right\|_{L^{1,2(\gamma+1)}}+\left\|U_{0}\right\|_{L^{1,2 \gamma+1}}\right) \\
& +C(1+t)^{-l / 2}\left\|\partial_{x}^{k+l} U_{0}\right\|_{2}
\end{aligned}
$$

where $k$ and $l$ are non-negative integers satisfying $k+l \leq s$ and $C$ and $c$ are two positive constants. This can be proved using [16, Lemma 2.3] and the estimate for the Fourier image, given in [38],

$$
|\hat{U}(\xi, t)|^{2} \leq C e^{-c \rho(\xi) t}|\hat{U}(\xi, 0)|^{2}
$$

where $\rho(\xi)=\xi^{2} /\left(1+\xi^{2}\right)^{2}$ and, C, c are two positive constants.

Now, we present the results on the global existence and on the asymptotic stability of the nonlinear problem (3.3). In order to state our main result, and led by [13], we introduce the time weighed energy norm $E(t)$ and the corresponding dissipation norm $D(t)$ as follows:

$$
E^{2}(t) \equiv \sum_{j=0}^{[s / 2]} \sup _{0 \leq \tau \leq t}(1+\tau)^{j-\frac{1}{2}}\left\|\partial_{x}^{j} U(\tau)\right\|_{H^{s-2 j}}^{2}
$$


and

$$
\begin{aligned}
D^{2}(t) \equiv & \sum_{j=0}^{[s / 2]} \int_{0}^{t}(1+\tau)^{j-\frac{3}{2}}\left\|\partial_{x}^{j} U(\tau)\right\|_{H^{s-2 j}}^{2} d \tau \\
& +\sum_{j=0}^{[s / 2]-1} \int_{0}^{t}(1+\tau)^{j-\frac{1}{2}}\left(\left\|\partial_{x}^{j} v(\tau)\right\|_{H^{s-1-2 j}}^{2}+\left\|\partial_{x}^{j} \theta_{x}(\tau)\right\|_{H^{s-2 j-1}}^{2}\right) d \tau \\
& +\sum_{j=0}^{[s / 2]} \int_{0}^{t}(1+\tau)^{j-\frac{1}{2}}\left(\left\|\partial_{x}^{j} y(\tau)\right\|_{H^{s-2 j}}^{2}+\left\|\partial_{x}^{j} w(\tau)\right\|_{H^{s-2 j}}^{2}\right) d \tau .
\end{aligned}
$$

Our main result reads as follows:

Theorem 3.3 Assume that $\sigma^{\prime}(\eta)>0$. Let $U_{0} \in H^{s}(\mathbb{R}) \cap L^{1}(\mathbb{R})$ with $s \geq 8$ and put $E_{0}:=$ $\left\|U_{0}\right\|_{H^{s}}+\left\|U_{0}\right\|_{L^{1}}$. Then, there exists a positive constant $\delta_{0}>0$ such that if $E_{0} \leq \delta_{0}$, then problem (3.3) has a unique global solution $U$ satisfying

$$
U \in C\left([0, \infty) ; H^{s}(\mathbb{R})\right) \cap C^{1}\left([0, \infty) ; H^{s-1}(\mathbb{R})\right)
$$

Moreover, the solution satisfies the weighted energy estimate

$$
E^{2}(t)+D^{2}(t) \leq C E_{0}^{2}
$$

and the decay estimate

$$
\left\|\partial_{x}^{k} U(t)\right\|_{2} \leq C E_{0}(1+t)^{-1 / 4-k / 2}
$$

where $C$ is a positive constant and $0 \leq k \leq[s / 2]-2$.

Remark 3.4 The assumption $U_{0} \in L^{1}(\mathbb{R})$ in Theorem 3.3 is needed only to prove the decay estimate (3.13). To prove the global existence, the requirement $U_{0} \in H^{s}(\mathbb{R})$ with the smallness assumption on $\left\|U_{0}\right\|_{H^{s}}$ is sufficient.

Remark 3.5 A similar result to Theorem 3.3 has been proved recently in [13] for the pure Timoshenko system (without heat conduction). Introducing the heat conduction of Cattaneo's law, renders the analysis more difficult. Moreover, for the pure Timoshenko system, the result of Theorem 3.3 holds for $s \geq 6$. Our requirement on $s$ here is $s \geq 8$, since it is not obvious to get the better decay estimate from [13, Corollary 2.1] in our case.

Remark 3.6 As we have explained in the introduction, if we consider the pure heat conduction of Cattaneo's law, we get the telegraph equation

$$
\left\{\begin{array}{l}
\rho_{3} \theta_{t t}-\frac{\varrho \kappa}{\tau_{0}} \Delta \theta+\frac{\rho_{3}}{\tau_{0}} \theta_{t}=0, \\
\theta(x, 0)=\theta_{0}(x), \quad \theta_{t}(x, 0)=\theta_{1}(x) .
\end{array}\right.
$$


The decay rate of (3.14) is of the form (see [20])

$$
\|\theta(t)\|_{2} \leq C(1+t)^{-N / 4}
$$

provided that $\left(\theta_{0}, \theta_{1}\right) \in\left(H^{1}\left(\mathbb{R}^{N}\right) \cap L^{1}\left(\mathbb{R}^{N}\right)\right) \times\left(L^{2}\left(\mathbb{R}^{N}\right) \cap L^{1}\left(\mathbb{R}^{N}\right)\right)$. Consequently, we conclude that the Timoshenko part is responsible for the regularity-loss property.

On the other hand, the Fourier law yields the parabolic equation

$$
\left\{\begin{array}{l}
\rho_{3} \tilde{\theta}_{t}-\kappa \varrho \Delta \tilde{\theta}=0, \\
\tilde{\theta}(x, 0)=\tilde{\theta}_{0} .
\end{array}\right.
$$

It is well known that the solution of (3.16) decays with the same rate as in (3.15) and the norm of the difference $\|\theta-\tilde{\theta}\|_{L^{2}}$ decays even better like $(1+t)^{-N / 4-1}$ if $\tilde{\theta}_{0}=\theta_{0}+\theta_{1}$. See [42]. Consequently, it is natural to expect that the characteristic behavior should be also the same in connection with elastic systems. However, this is not always the case. Recently it has been proved in [8] that for the Timoshenko systems in bounded domain, exponential stability is lost when substituting the Fourier law of heat conduction by Cattaneo's law, and the behavior of the two systems are different. The same conclusion holds for the coupling with the plate equation [30].

Remark 3.7 The smallness assumption on the initial data in Theorem 3.3 seems necessary to prove the global existence of solutions. Globally defined solution should not be expected for large initial data. This is still an interesting open problem. In fact this is also an open problem even for the Cauchy problem associated to system (1.8). For the Cauchy problem in classical thermoelasticity, Hrusa and Messaoudi [11] have shown that if the initial data are large enough, then the solution will develop singularities in finite time. (See also [7] for a similar result).

To prove Theorem 3.3, we use the same method as in [13] with the necessary modifications imposed by the nature of our problem. The proof will be a direct consequence of Lemma 3.8 .

Let us first define the quantities

$$
\begin{aligned}
& M_{0}(t):=\sup _{0 \leq \tau \leq t}(1+\tau)^{\frac{1}{2}}\|U(\tau)\|_{L^{\infty}}, \\
& M_{1}(t):=\sup _{0 \leq \tau \leq t}(1+\tau)\left\|\partial_{x} U(\tau)\right\|_{L^{\infty}} .
\end{aligned}
$$

and inspired by the estimates (3.5), we define

$$
M(t):=\sum_{j=0}^{[s / 2]-2} \sup _{0 \leq \tau \leq t}(1+\tau)^{1 / 4+j / 2}\left\|\partial_{x}^{j} U(\tau)\right\|_{2} .
$$

We have the following Lemma. 
Lemma 3.8 Assume that the conditions of Theorem 3.3 hold. Let $T>0$ and $s \geq 2$ and let $U$ be a solution to the problem (3.3) satisfying

$$
U \in C\left([0, T] ; H^{s}(\mathbb{R}) \cap C^{1}(0, T] ; H^{s-1}(\mathbb{R}) .\right.
$$

Then we have the a priori estimates

$$
\begin{aligned}
E^{2}(T)+D^{2}(T) & \leq C E_{0}^{2} \\
M(T) & \leq C E_{0}
\end{aligned}
$$

where $E_{0}$ is given in Theorem 3.3 and $C$ is a positive constant independent of $T$.

The local existence theorem needed in Lemma 3.8 can be proved by standard methods for symmetric-hyperbolic systems, see subsection 4.3. The global existence is based on a priori estimates that can be used to continue a local solution globally in time.

So, let $T>0$ and consider solutions to the problem (3.3) which are defined on the time interval $[0, T]$ and satisfy the regularity mentioned in Lemma 3.8.

Thanks to the assumption $s>\frac{N}{2}+1$ (where $N$ is the space dimension which is one in our case), it follows from the Sobolev embedding theorem that

$$
\sup _{0 \leq \tau \leq t}\|U(\tau)\|_{L^{\infty}}+\sup _{0 \leq \tau \leq t}\left\|\partial_{x} U(\tau)\right\|_{L^{\infty}} \leq C\|U(t)\|_{H^{s}} .
$$

We shall derive the energy estimates under the a priori assumption

$$
\sup _{0 \leq t \leq T}\|U(t)\|_{L^{\infty}} \leq \bar{\alpha}
$$

where $\bar{\alpha}$ is a fixed small number, independent of $T$.

In order to prove Lemma 3.8, we have to use Proposition 3.9 and Lemma 3.10 below. Proposition 3.9, will be proved in subsection 4.1, while subsection 4.2 is devoted to the proof of Lemma 3.10. Lemma 3.8 and Theorem 3.3 will be proved in subsection 4.3.

Proposition 3.9 Suppose that the assumptions in Theorem 3.3 hold. Let $T>0$ and $s \geq 2$, and let $U$ be the solution of problem (3.1)-(3.2) satisfying (3.11) and (3.19). Then, the estimate

$$
E(t)^{2}+D(t)^{2} \leq C\left\|U_{0}\right\|_{H^{s}}^{2}+C\left(M_{0}(t)+M_{1}(t)\right) D^{2}(t),
$$

holds true for all $t \in[0, T]$, where $C$ is a positive constant which is independent of $T$.

Lemma 3.10 Under the same assumptions as in Proposition 3.9, and supposing that $U_{0} \in$ $H^{s}(\mathbb{R}) \cap L^{1}(\mathbb{R})$ with $s \geq 2$, we have

$$
M(t) \leq C E_{0}+C M(t)^{2}+C M_{0}(t) E(t)
$$

for all $t \in[0, T]$, where $C$ is a positive constant which is independent of $T$, and $E_{0}$ is given in Theorem 3.3. 


\section{Proof of the main results}

In this section, we prove our main results presented in section 3.

\subsection{Proof of Proposition 3.9}

We proceed with the basic energy estimate by multiplying the first equation in (3.1) by $v$, the second equation by $u$, the third by $(\sigma(z / a)-\sigma(0)) / a$, the fourth by $y$, the fifth $\theta$ and the sixth equation by $\frac{1}{\tau_{0}} w$, respectively, adding the resulting equations, and integrating with respect to $x$ over $\mathbb{R}$, we obtain

$$
\frac{d}{d t} E^{(0)}(t)+\alpha\|y\|_{2}^{2}+\frac{\delta}{\tau_{0}^{2}}\|w\|_{2}^{2}=0
$$

where

$$
E^{(0)}(t):=\frac{1}{2}\left(\|v\|_{2}^{2}+\|u\|_{2}^{2}+\|y\|_{2}^{2}+\frac{1}{\tau_{0}}\|w\|_{2}^{2}+\|\theta\|_{2}^{2}\right)+\int_{\mathbb{R}} F(z) d x
$$

and

$$
F(z):=2 \int_{0}^{z / a}(\sigma(s)-\sigma(0)) d s
$$

To obtain the energy estimates on higher-order terms, applying, for $k \geq 1, \partial_{x}^{k}$ to (3.1), we get

$$
\left\{\begin{array}{l}
\partial_{x}^{k} v_{t}-\partial_{x}^{k+1} u+\partial_{x}^{k} y=0 \\
\partial_{x}^{k} u_{t}-\partial_{x}^{k+1} v=0 \\
\partial_{x}^{k} z_{t}-a \partial_{x}^{k+1} y=0 \\
\partial_{x}^{k} y_{t}-\sigma^{\prime}(z / a) \partial_{x}^{k+1}(z / a)-\partial_{x}^{k} v+\alpha \partial_{x}^{k} y+\beta \partial_{x}^{k+1} \theta=\left[\partial_{x}^{k}, \sigma^{\prime}(z / a)\right](z / a)_{x}, \\
\partial_{x}^{k} \theta_{t}+\frac{\kappa}{\tau_{0}} \partial_{x}^{k+1} w+\beta \partial_{x}^{k+1} y=0 \\
\partial_{x}^{k} w_{t}+\frac{\delta}{\tau_{0}} \partial_{x}^{k} w+\kappa \partial_{x}^{k+1} \theta=0
\end{array}\right.
$$

where we have used the notation $\left[\partial_{x}^{k}, A\right] B:=\partial_{x}^{k}(A B)-A \partial_{x}^{k} B$.

Now, define the energy associated to system (4.3) as

$$
E^{k}(t):=\frac{1}{2}\left(\left\|\partial_{x}^{k} v\right\|_{2}^{2}+\left\|\partial_{x}^{k} u\right\|_{2}^{2}+\left\|\partial_{x}^{k} y\right\|_{2}^{2}+\left\|\partial_{x}^{k} w\right\|_{2}^{2}+\frac{1}{\tau_{0}}\left\|\partial_{x}^{k} \theta\right\|_{2}^{2}\right)+\int_{\mathbb{R}} F^{k}(z) d x
$$

where

$$
F^{k}(z):=\frac{1}{2} \sigma^{\prime}(z / a)\left(\partial_{x}^{k} z / a\right)^{2} .
$$

Now, we try to find a Lyapunov functional and appropriate multipliers. To do so, some nonlinear terms may arise and we treat these non-linear terms as perturbations of the energy 
terms. The proof somehow imitates the energy estimates in the Fourier space derived for the linear problem in [38].

Thus, multiplying the first equation in (4.3) by $\partial_{x}^{k} v$, the second equation by $\partial_{x}^{k} u$, the third by $\left(1 / a^{2}\right)\left(\sigma^{\prime}(z / a) \partial_{x}^{k} z\right)$, the fourth by $\partial_{x}^{k} y$, the fifth equation by $\partial_{x}^{k} \theta$ and the sixth equation by $\frac{1}{\tau_{0}} \partial_{x}^{k} w$, respectively, adding the resulting equations, and integrating with respect to $x$, we obtain

$$
\frac{d}{d t} E^{(k)}(t)+\alpha\left\|\partial_{x}^{k} y\right\|_{2}^{2}+\frac{\delta}{\tau_{0}^{2}}\left\|\partial_{x}^{k} w\right\|_{2}^{2}=R_{0}^{(k)}
$$

where

$$
R_{0}^{(k)}:=\int_{\mathbb{R}}\left\{\frac{1}{2} \sigma^{\prime}(z / a)_{t}\left(\partial_{x}^{k} z / a\right)^{2}-\sigma^{\prime}(z / a)_{x}\left(\partial_{x}^{k} z / a\right) \partial_{x}^{k} y+\partial_{x}^{k} y\left[\partial_{x}^{k}, \sigma^{\prime}(z / a)\right](z / a)_{x}\right\} d x .
$$

Using the assumption (3.19) and as in [13], we get

$$
\left|R_{0}^{(k)}\right| \leq C \int_{\mathbb{R}}\left|y_{x}\right|\left|\partial_{x}^{k} z\right|^{2}+\left|z_{x}\right|\left|\partial_{x}^{k} z\right|\left|\partial_{x}^{k} y\right|+\left|\partial_{x}^{k} y\right|\left|\left[\partial_{x}^{k}, \sigma^{\prime}(z / a)\right]\right|\left|z_{x}\right|,
$$

where $C=C(\bar{\alpha})$ with $\bar{\alpha}$ from (3.19). This implies that, by using Lemma 2.2 (see [13] for details),

$$
\left|R_{0}^{(k)}\right| \leq C\left\|\partial_{x} U\right\|_{L^{\infty}}\left\|\partial_{x}^{k} U\right\|_{L^{2}}^{2}
$$

On the other hand, recalling (3.19), we deduce that there exist two positive constants $\beta_{1}$ and $\beta_{2}$ depending on $\bar{\alpha}$, such that

$$
\beta_{1}\left\|\partial_{x}^{k} U\right\|_{L^{2}}^{2} \leq E^{k}(t) \leq \beta_{2}\left\|\partial_{x}^{k} U\right\|_{L^{2}}^{2}, \quad k \geq 0 .
$$

Consequently, multiplying (4.1) by $(1+t)^{\mu}$, with $\mu \in \mathbb{R}$ (later to be chosen as $\mu=-1 / 2$ ), and integrating with respect to $t$ and using (4.7) we get

$$
\begin{aligned}
& (1+t)^{\mu}\|U(t)\|_{L^{2}}^{2}+\alpha \int_{0}^{t}(1+s)^{\mu}\|y(s)\|_{2}^{2} d s+\frac{\delta}{\tau_{0}^{2}} \int_{0}^{t}(1+s)^{\mu}\|w(s)\|_{2}^{2} d s \\
\leq & \left\|U_{0}\right\|_{L^{2}}^{2}+\mu \int_{0}^{t}(1+s)^{\mu-1}\|U(s)\|_{L^{2}}^{2} d s .
\end{aligned}
$$

Similarly, for $k \geq 1$, the estimates (4.6), (4.7) together with (4.5) yield, after a multiplication by $(1+t)^{\mu}$ and integration with respect to $t$ over $(0, t)$

$$
\begin{aligned}
& (1+t)^{\mu}\left\|\partial_{x}^{k} U(t)\right\|_{L^{2}}^{2}+\alpha \int_{0}^{t}(1+s)^{\mu}\left\|\partial_{x}^{k} y(s)\right\|_{2}^{2} d s \\
& +\frac{\delta}{\tau_{0}^{2}} \int_{0}^{t}(1+s)^{\mu}\left\|\partial_{x}^{k} w(s)\right\|_{2}^{2} d s \\
& \leq C\left\|\partial_{x}^{k} U_{0}\right\|_{L^{2}}^{2}+\mu \int_{0}^{t}(1+s)^{\mu-1}\left\|\partial_{x}^{k} U(s)\right\|_{L^{2}}^{2} d s \\
& +C \int_{0}^{t}(1+s)^{\mu}\left\|\partial_{x} U(s)\right\|_{L^{\infty}}\left\|\partial_{x}^{k} U(s)\right\|_{L^{2}}^{2} d s .
\end{aligned}
$$


Adding the estimate (4.8) to (4.9) and taking the summation for $1 \leq k \leq s$, we get the main estimate

$$
\begin{aligned}
& (1+t)^{\mu}\|U(t)\|_{H^{s}}^{2}+\alpha \int_{0}^{t}(1+s)^{\mu}\|y(s)\|_{H^{s}}^{2} d s \\
& +\frac{\delta}{\tau_{0}^{2}} \int_{0}^{t}(1+s)^{\mu}\|w(s)\|_{H^{s}}^{2} d s \\
& \leq C\left\|U_{0}\right\|_{H^{s}}^{2}+\mu \int_{0}^{t}(1+s)^{\mu-1}\|U(s)\|_{H^{s}}^{2} d s \\
& +C \int_{0}^{t}(1+s)^{\mu}\left\|\partial_{x} U(s)\right\|_{L^{\infty}}\left\|\partial_{x} U(s)\right\|_{H^{s-1}}^{2} d s .
\end{aligned}
$$

Our goal now is to control the second term in the right-hand side of (4.10). To do so, we have to get a dissipative term of the form $\int_{0}^{t}\|U(t)\|_{H^{s}}^{2}$ on the left-hand side of (4.10).

Applying $\partial_{x}^{k}$ to system (3.1) and put $\partial_{x}^{k}(u, v, z, y, \theta, w)=(\tilde{u}, \tilde{v}, \tilde{z}, \tilde{y}, \tilde{\theta}, \tilde{w})$, system (3.1) can be rewritten as

$$
\left\{\begin{array}{l}
\tilde{v}_{t}-\tilde{u}_{x}+\tilde{y}=0 \\
\tilde{u}_{t}-\tilde{v}_{x}=0 \\
\tilde{z}_{t}-a \tilde{y}_{x}=0 \\
\tilde{y}_{t}-a \tilde{z}_{x}-\tilde{v}+\alpha \tilde{y}+\beta \tilde{\theta}_{x}=\partial_{x}^{k} g(z)_{x} \\
\tilde{\theta}_{t}+\frac{\kappa}{\tau_{0}} \tilde{w}_{x}+\beta \tilde{y}_{x}=0 \\
\tilde{w}_{t}+\frac{\delta}{\tau_{0}} \tilde{w}+\kappa \tilde{\theta}_{x}=0
\end{array}\right.
$$

where $g(z):=\sigma(z / a)-\sigma(0)-\sigma^{\prime}(0) z / a=O\left(z^{2}\right)$ near $z=0$.

In the calculations that follow, we make repeated use of the Young inequality

$$
|d b| \leq \epsilon d^{2}+C(\epsilon) b^{2},
$$

where $C(\epsilon)$ here and in the sequel will denote possibly different values in different places (and can be easily determined explicitly).

First, multiplying the first equation in (4.11) by $-\tilde{u}_{x}$, the second equation by $\tilde{v}_{x}$ and adding the resulting equations, we get

$$
-\left(\tilde{v} \tilde{u}_{x}\right)_{t}+\left(\tilde{v} \tilde{u}_{t}\right)_{x}+\tilde{u}_{x}^{2}-\tilde{v}_{x}^{2}-\tilde{u}_{x} \tilde{y}=0 .
$$

Similarly, multiplying the third equation in (4.11) by $\tilde{y}_{x}$, and the fourth equation by $\tilde{z}_{x}$, and subtracting the two equations, we obtain

$$
\left(\tilde{z}_{t} \tilde{y}\right)_{x}-\left(\tilde{y} \tilde{z}_{x}\right)_{t}+a \tilde{z}_{x}^{2}-a \tilde{y}_{x}^{2}+(\tilde{v}-\alpha \tilde{y}) \tilde{z}_{x}-\beta \tilde{\theta}_{x} \tilde{z}_{x}=-\tilde{z}_{x} \partial_{x}^{k} g(z)_{x}
$$

Now, we add the equalities (4.12) and (4.13), and we obtain

$$
\begin{aligned}
& -\left(\tilde{v} \tilde{u}_{x}+\tilde{y} \tilde{z}_{x}\right)_{t}+\left(\tilde{v} \tilde{u}_{t}+\tilde{z}_{t} \tilde{y}\right)_{x}+\tilde{u}_{x}^{2}+a \tilde{z}_{x}^{2} \\
& =\tilde{v}_{x}^{2}+a \tilde{y}_{x}^{2}+\tilde{u}_{x} \tilde{y}-(\tilde{v}-\alpha \tilde{y}) \tilde{z}_{x}+\beta \tilde{\theta}_{x} \tilde{z}_{x}-\tilde{z}_{x} \partial_{x}^{k} g(z)_{x} .
\end{aligned}
$$


Using Young's inequality, we get for any $\epsilon>0$

$$
\tilde{u}_{x} \tilde{y}-(\tilde{v}-\alpha \tilde{y}) \tilde{z}_{x} \leq \epsilon\left(\tilde{u}_{x}^{2}+\tilde{z}_{x}^{2}\right)+C(\epsilon)\left(\tilde{v}^{2}+\tilde{y}^{2}\right)
$$

and

$$
\beta \tilde{\theta}_{x} \tilde{z}_{x} \leq \epsilon \tilde{z}_{x}^{2}+C(\epsilon) \tilde{\theta}_{x}^{2} .
$$

Now, plugging the inequalities (4.15) and (4.16) into (4.14), and integrating the result with respect to $x$, we get

$$
\begin{aligned}
& \frac{d \mathscr{F}^{(k)}(t)}{d t}+(1-\epsilon)\left\|\partial_{x}^{k} u_{x}\right\|_{2}^{2}+(a-2 \epsilon)\left\|\partial_{x}^{k} z_{x}\right\|_{2}^{2} \\
& \leq C(\epsilon)\left(\left\|\partial_{x}^{k} v\right\|_{H^{1}}^{2}+\left\|\partial_{x}^{k} y\right\|_{H^{1}}^{2}\right)+C(\epsilon)\left\|\partial_{x}^{k} \theta_{x}\right\|_{2}^{2}+R_{1}^{(k)}
\end{aligned}
$$

where

$$
\mathscr{F}^{(k)}(t):=-\int_{\mathbb{R}}\left(\partial_{x}^{k} v \partial_{x}^{k} u_{x}+\partial_{x}^{k} y \partial_{x}^{k} z_{x}\right) d x, \quad R_{1}^{(k)}:=\int_{\mathbb{R}}\left|\partial_{x}^{k} z_{x}\right|\left|\partial_{x}^{k} g(z)_{x}\right| d x .
$$

Also, multiplying the fifth equation in $(4.11)$ by $\tilde{w}_{x}$, and the sixth equation by $\tilde{\theta}_{x}$ and subtracting the two resulting equations, we find

$$
\left(-\tilde{\theta} \tilde{w}_{x}\right)_{t}+\left(\tilde{w}_{t} \tilde{\theta}\right)_{x}+\kappa \tilde{\theta}_{x}^{2}-\frac{\kappa}{\tau_{0}} \tilde{w}_{x}^{2}-\beta \tilde{y}_{x} \tilde{w}_{x}+\frac{\delta}{\tau_{0}} \tilde{w} \tilde{\theta}_{x}=0 .
$$

Young's inequality gives

$$
\beta \tilde{y}_{x} \tilde{w}_{x}+\frac{\delta}{\tau_{0}} \tilde{w} \tilde{\theta}_{x} \leq \epsilon\left(\tilde{y}_{x}^{2}+\tilde{\theta}_{x}^{2}\right)+C(\epsilon)\left(\tilde{w}^{2}+\tilde{w}_{x}^{2}\right) .
$$

Now, inserting the inequality (4.20) into (4.19) and integrating the result with respect to $x$, we get

$$
\frac{d}{d t} \mathscr{N}^{(k)}(t)+(\kappa-\epsilon)\left\|\partial_{x}^{k} \theta_{x}\right\|_{2}^{2} \leq \epsilon\left\|\partial_{x}^{k} y_{x}\right\|_{2}^{2}+C(\epsilon)\left(\left\|\partial_{x}^{k} w\right\|_{2}^{2}+\left\|\partial_{x}^{k} w_{x}\right\|_{2}^{2}\right)
$$

where

$$
\mathscr{N}^{(k)}(t):=-\int_{\mathbb{R}} \partial_{x}^{k} \theta \partial_{x}^{k} w_{x} d x
$$

Next, multiplying the first equation in (4.11) by $-\tilde{y}$ and the fourth equation by $-\tilde{v}$ and adding the two results, we obtain

$$
-(\tilde{v} \tilde{y})_{t}+\tilde{v}^{2}-\tilde{y}^{2}+\tilde{u}_{x} \tilde{y}+a \tilde{z}_{x} \tilde{v}-\alpha \tilde{y} \tilde{v}-\beta \tilde{\theta}_{x} \tilde{v}=-\tilde{v} \partial_{x}^{k} g(z)_{x} .
$$

Similarly, multiplying the second equation in (4.11) by $-a \tilde{z}$, the third equation by $-a \tilde{u}$ and adding the two equations, we find

$$
-(a \tilde{z} \tilde{u})_{t}+a \tilde{z} \tilde{v}_{x}+a^{2} \tilde{u} \tilde{y}_{x}=0 .
$$


Subtracting the equation (4.23) from (4.22), we find

$$
-(\tilde{v} \tilde{y}-a \tilde{z} \tilde{u})_{t}-a(\tilde{u} \tilde{y})_{x}+\tilde{v}^{2}-\tilde{y}^{2}=\left(\left(1-a^{2}\right) \tilde{u}_{x} \tilde{y}+\alpha \tilde{y} \tilde{v}+\beta \tilde{\theta}_{x} \tilde{v}\right)-\tilde{v} \partial_{x}^{k} g(z)_{x} .
$$

Applying Young's inequality to the first term in the right-hand side of (4.24), we obtain for any $\epsilon>0$

$$
\left(\alpha \tilde{y} \tilde{v}+\beta \tilde{\theta}_{x} \tilde{v}\right) \leq \epsilon \tilde{v}^{2}+C(\epsilon)\left(\tilde{y}^{2}+\tilde{\theta}_{x}^{2}\right) .
$$

Consequently, inserting (4.25) into (4.24) and integrating the result with respect to the $x$ variable, we get

$$
\begin{aligned}
& \frac{d}{d t} \mathscr{K}^{(k)}(t)+(1-\epsilon)\left\|\partial_{x}^{k} v\right\|_{2}^{2} \\
& \leq C(\epsilon)\left(\left\|\partial_{x}^{k} y\right\|_{2}^{2}+\left\|\partial_{x}^{k} \theta_{x}\right\|_{2}^{2}\right)+\left(1-a^{2}\right) \int_{\mathbb{R}} \tilde{u}_{x} \tilde{y} d x+R_{2}^{(k)}
\end{aligned}
$$

where

$$
\mathscr{K}^{(k)}(t):=\int_{\mathbb{R}}\left(-\partial_{x}^{k} v \partial_{x}^{k} y+a \partial_{x}^{k} z \partial_{x}^{k} u\right) d x \quad R_{2}^{(k)}:=\int_{\mathbb{R},}\left|\partial_{x}^{k} v\right|\left|\partial_{x}^{k} g(z)_{x}\right| .
$$

Of course, the above estimates (4.17), (4.21) and (4.26) hold for every $0 \leq k \leq s-1$.

It is clear that from (4.17), (4.21) and (4.26) we get, for any $0 \leq k \leq s-2$

$$
\begin{aligned}
& \frac{d}{d t} \mathscr{L}^{(k)}(t)+\left\{\alpha_{2}(\kappa-\epsilon)-\alpha_{1} C(\epsilon)-\alpha_{3} C(\epsilon)\right\}\left\|\partial_{x}^{k} \theta_{x}\right\|_{H^{1}}^{2}+\alpha_{1}(a-2 \epsilon)\left\|\partial_{x}^{k} z_{x}\right\|_{2}^{2} \\
& +\left\{\alpha_{3}(1-\epsilon)-\alpha_{1} C(\epsilon)\right\}\left\|\partial_{x}^{k} v\right\|_{H^{1}}^{2}+\alpha_{1}(1-\epsilon)\left\|\partial_{x}^{k} u_{x}\right\|_{2}^{2} \\
\leq & \left\{\alpha_{1} C(\epsilon)+\alpha_{3} C(\epsilon)+\alpha_{2} \epsilon\right\}\left\|\partial_{x}^{k} y\right\|_{H^{2}}^{2}+\alpha_{2} C(\epsilon)\left\|\partial_{x}^{k} w\right\|_{H^{2}}^{2} \\
& +\alpha_{3}\left(1-a^{2}\right) \int_{\mathbb{R}}\left(\partial_{x}^{k} u_{x} \partial_{x}^{k} y+\partial_{x}^{k+1} u_{x} \partial_{x}^{k+1} y\right) d x+R_{1}^{(k)}+\alpha_{3}\left(R_{2}^{(k)}+R_{2}^{(k+1)}\right)
\end{aligned}
$$

where

$$
\mathscr{L}^{(k)}(t)=\mathscr{F}^{(k)}(t)+\alpha_{2}\left(\mathscr{N}^{(k)}(t)+\mathscr{N}^{(k+1)}(t)\right)+\alpha_{3}\left(\mathscr{K}^{(k)}(t)+\mathscr{K}^{(k+1)}(t)\right),
$$

and $\alpha_{2}, \alpha_{3}>0$ have to be determined appropriately below. The last integral term in (4.28) can be estimated as follows:

$$
\begin{aligned}
& \left(1-a^{2}\right) \int_{\mathbb{R}}\left(\partial_{x}^{k} u_{x} \partial_{x}^{k} y+\partial_{x}^{k+1} u_{x} \partial_{x}^{k+1} y\right) d x \\
= & \left(1-a^{2}\right) \int_{\mathbb{R}} \partial_{x}^{k} u_{x}\left(\partial_{x}^{k} y-\partial_{x}^{k} y_{x x}\right) d x \\
\leq & \tilde{\epsilon}\left\|\partial_{x}^{k} u_{x}\right\|_{2}^{2}+C(\tilde{\epsilon})\left\|\partial_{x}^{k} y\right\|_{H^{2}}^{2} .
\end{aligned}
$$


Plugging the estimate (4.29) into (4.28), we find

$$
\begin{aligned}
& \frac{d}{d t} \mathscr{L}^{(k)}(t)+\left\{\alpha_{2}(\kappa-\epsilon)-\alpha_{1} C(\epsilon)-\alpha_{3} C(\epsilon)\right\}\left\|\partial_{x}^{k} \theta_{x}\right\|_{H^{1}}^{2}+\alpha_{1}(a-2 \epsilon)\left\|\partial_{x}^{k} z_{x}\right\|_{2}^{2} \\
& +\left\{\alpha_{3}(1-\epsilon)-\alpha_{1} C(\epsilon)\right\}\left\|\partial_{x}^{k} v\right\|_{H^{1}}^{2}+\left\{\alpha_{1}(1-\epsilon)-\alpha_{3} \tilde{\epsilon}\right\}\left\|\partial_{x}^{k} u_{x}\right\|_{2}^{2} \\
\leq & \left\{\alpha_{1} C(\epsilon)+\alpha_{3} C(\epsilon)+\alpha_{2} \epsilon+\alpha_{3} C(\tilde{\epsilon})\right\}\left\|\partial_{x}^{k} y\right\|_{H^{2}}^{2}+\alpha_{2} C(\epsilon)\left\|\partial_{x}^{k} w\right\|_{H^{2}}^{2} \\
& +R_{1}^{(k)}+\alpha_{3}\left(R_{2}^{(k)}+R_{2}^{(k+1)}\right) .
\end{aligned}
$$

Now, we fix the constants in the above estimate as follows: First, we fix $\epsilon$ small enough such that

$$
\epsilon \leq \min \left(\frac{\kappa}{2}, 1, \frac{a}{4}\right) .
$$

After that, we choose $\alpha_{2}$ and $\alpha_{3}$ large enough such that

$$
\left\{\begin{array}{l}
\alpha_{3}(1-\epsilon)-C(\epsilon)>0 \\
\alpha_{2} \frac{\kappa}{2 \tau_{0}}-C(\epsilon)-\alpha_{3} C(\epsilon)>0
\end{array}\right.
$$

Once, the above constants are fixed, we choose $\tilde{\epsilon}$ small enough such that

$$
(1-\epsilon)-\alpha_{3} \tilde{\epsilon}>0
$$

Consequently, for any $0 \leq k \leq s-2$, the estimate (4.30) takes the form

$$
\begin{aligned}
& \frac{d}{d t} \mathscr{L}^{(k)}(t)+c_{1}\left(\left\|\partial_{x}^{k} \theta_{x}\right\|_{H^{1}}^{2}+\left\|\partial_{x}^{k} z_{x}\right\|_{2}^{2}+\left\|\partial_{x}^{k} v\right\|_{H^{1}}^{2}+\left\|\partial_{x}^{k} u_{x}\right\|_{2}^{2}\right) \\
\leq & c_{2}\left(\left\|\partial_{x}^{k} y\right\|_{H^{2}}^{2}+\left\|\partial_{x}^{k} w\right\|_{H^{2}}^{2}\right)+R_{1}^{(k)}+\alpha_{3}\left(R_{2}^{(k)}+R_{2}^{(k+1)}\right),
\end{aligned}
$$

where $c_{1}$ and $c_{2}$ are two positive constants.

Thus, the estimate (4.31) can be written as

$$
\begin{aligned}
& \frac{d}{d t} \mathscr{L}^{(k)}(t)+c_{3}\left(\left\|\partial_{x}^{k} \theta_{x}\right\|_{H^{1}}^{2}+\left\|\partial_{x}^{k+1} U\right\|_{2}^{2}+\left\|\partial_{x}^{k} v\right\|_{H^{1}}^{2}\right) \\
\leq & \tilde{c}_{2}\left(\left\|\partial_{x}^{k} y\right\|_{H^{2}}^{2}+\left\|\partial_{x}^{k} w\right\|_{H^{2}}^{2}\right)+R_{1}^{(k)}+\alpha_{3}\left(R_{2}^{(k)}+R_{2}^{(k+1)}\right), \quad \forall t \geq 0,
\end{aligned}
$$

where $\tilde{c}_{2}$ and $c_{3}$ are two positive constants. On the other hand, it is easy to see that there exists a constant $c_{4}>0$, such that

$$
\left|\mathscr{L}^{(k)}(t)\right| \leq c_{4}\left\|\partial_{x}^{k} U(t)\right\|_{H^{2}}^{2}, \quad \forall t \geq 0 .
$$

Also, as in [13], we have the following estimates

$$
R_{1}^{(k)} \leq C\|z\|_{L^{\infty}}\left\|\partial_{x}^{k+1} z\right\|_{2}^{2}, \quad R_{2}^{(k)} \leq C\|z\|_{L^{\infty}}\left\|\partial_{x}^{k} v\right\|_{2}\left\|\partial_{x}^{k+1} z\right\|_{2} .
$$


Now, multiplying (4.32) by $(1+t)^{\mu}$ and integrating with respect to $t$ and exploiting (4.33) and (4.34), we arrive at

$$
\begin{aligned}
& \int_{0}^{t}(1+s)^{\mu}\left(\left\|\partial_{x}^{k} \theta_{x}(s)\right\|_{H^{1}}^{2}+\left\|\partial_{x}^{k+1} U(s)\right\|_{2}^{2}+\left\|\partial_{x}^{k} v(s)\right\|_{H^{1}}^{2}\right) d s \\
\leq & C\left\|\partial_{x}^{k} U_{0}\right\|_{H^{2}}^{2}+C(1+t)^{\mu}\left\|\partial_{x}^{k} U(t)\right\|_{H^{2}}^{2}+C \mu \int_{0}^{t}(1+s)^{\mu-1}\left\|\partial_{x}^{k} U(s)\right\|_{H^{2}}^{2} d s \\
& +C \int_{0}^{t}(1+s)^{\mu}\left(\left\|\partial_{x}^{k} y(s)\right\|_{H^{2}}^{2}+\left\|\partial_{x}^{k} w(s)\right\|_{H^{2}}^{2}\right) d s \\
& +C \int_{0}^{t}(1+s)^{\mu}\|z\|_{L^{\infty}}\left(\left\|\partial_{x}^{k+1} z(s)\right\|_{2}^{2}+\left\|\partial_{x}^{k} v(s)\right\|_{2}\left\|\partial_{x}^{k+1} z(s)\right\|_{2}\right. \\
& \left.+\left\|\partial_{x}^{k+1} v(s)\right\|_{2}\left\|\partial_{x}^{k+2} z(s)\right\|_{2}\right) d s
\end{aligned}
$$

for all $t \geq 0$ and for $0 \leq k \leq s-2$. Taking the summation in (4.35) over $k$ with $0 \leq k \leq s-2$, we get

$$
\begin{aligned}
& \int_{0}^{t}(1+s)^{\mu}\left(\left\|\partial_{x} \theta(s)\right\|_{H^{s-1}}^{2}+\left\|\partial_{x} U(s)\right\|_{H^{s-2}}^{2}+\left\|\partial_{x}^{k} v(s)\right\|_{H^{s-1}}^{2}\right) d s \\
\leq & C\left\|U_{0}\right\|_{H^{s}}^{2}+C(1+t)^{\mu}\|U(t)\|_{H^{s}}^{2}+C \mu \int_{0}^{t}(1+s)^{\mu-1}\|U(s)\|_{H^{s}}^{2} d s \\
& +C \int_{0}^{t}(1+s)^{\mu}\left(\|y(s)\|_{H^{s}}^{2}+\|w(s)\|_{H^{s}}^{2}\right) d s \\
& +C \int_{0}^{t}(1+s)^{\mu}\|z\|_{L^{\infty}}\left(\left\|\partial_{x} z(s)\right\|_{H^{s-2}}^{2}+\|v(s)\|_{H^{s-1}}\left\|\partial_{x} z(s)\right\|_{H^{s-1}}\right) d s .
\end{aligned}
$$

Now, let $\lambda>0$ be a small positive constant, then computing (4.10) $+\lambda(4.36)$ and choosing $\lambda$ small enough, we arrive at the following estimate:

$$
\begin{aligned}
& (1+t)^{\mu}\|U(t)\|_{H^{s}}^{2}+\int_{0}^{t}(1+s)^{\mu}\left(\|y(s)\|_{H^{s}}^{2}+\|w(s)\|_{H^{s}}^{2}+\left\|\partial_{x} \theta(s)\right\|_{H^{s-1}}^{2}\right) d s \\
& +\int_{0}^{t}(1+s)^{\mu}\left(\left\|\partial_{x} U(s)\right\|_{H^{s-2}}^{2}+\|v(s)\|_{H^{s-1}}^{2}\right) d s \\
& \leq C\left\|U_{0}\right\|_{H^{s}}^{2}+C \mu \int_{0}^{t}(1+s)^{\mu-1}\|U(s)\|_{H^{s}}^{2} d s \\
& +C \int_{0}^{t}(1+s)^{\mu}\left\|\partial_{x} U(s)\right\|_{L^{\infty}}\left\|\partial_{x} U(s)\right\|_{H^{s-1}}^{2} d s \\
& +C \lambda \int_{0}^{t}(1+s)^{\mu}\|z\|_{L^{\infty}}\left(\left\|\partial_{x} z(s)\right\|_{H^{s-2}}^{2}+\|v(s)\|_{H^{s-1}}\left\|\partial_{x} z(s)\right\|_{H^{s-1}}\right) d s
\end{aligned}
$$

where $C$ is a generic positive constant depending on $\lambda$. 
It is obvious from the estimate (4.37), and for $\mu=0$, that the term $\int_{0}^{t}\left\|\partial_{x} U(s)\right\|_{H^{s-2}}^{2} d s$ on the left-hand side of (4.37) is not enough to control $\int_{0}^{t}\left\|\partial_{x} U(s)\right\|_{L^{\infty}}\left\|\partial_{x} U(s)\right\|_{H^{s-1}}^{2} d s$ appearing on the right-hand side of (4.37). Indeed, classical methods suggest that the nonlinearity in (4.37) can be estimates as (for example)

$$
\int_{0}^{t}\left\|\partial_{x} U(s)\right\|_{L^{\infty}}\left\|\partial_{x} U(s)\right\|_{H^{s-1}}^{2} d s \leq \sup _{0 \leq s \leq t}\|\partial U(s)\|_{L^{\infty}} \int_{0}^{t}\left\|\partial_{x} U(s)\right\|_{H^{s-1}}^{2} d s .
$$

However, the term $\int_{0}^{t}\left\|\partial_{x} U(s)\right\|_{H^{s-1}}^{2} d s$ on the right-hand side can not be controlled by $\int_{0}^{t}\left\|\partial_{x} U(s)\right\|_{H^{s-2}}^{2} d s$ on the left-hand side. To overcome this difficulty, we have to use the time weighted energy method with negative weights. That is, we have to take $\mu<0$ in (4.37). (see also [10,13] and [15] for more details). This procedure allows us to get the term $\mu \int_{0}^{t}(1+t)^{\mu-1}\|U(s)\|_{H^{s}}^{2} d s$, which can control the term $\int_{0}^{t}(1+t)^{\mu}\left\|\partial_{x} U(s)\right\|_{H^{s-1}}^{2} d s$ as long as $\mu<0$.

For $\lambda$ sufficiently small and for $\mu=-1 / 2$, the estimate (4.37) takes the form

$$
\begin{aligned}
& (1+t)^{-1 / 2}\|U(t)\|_{H^{s}}^{2}+\int_{0}^{t}(1+s)^{-1 / 2}\left(\|y(s)\|_{H^{s}}^{2}+\|w(s)\|_{H^{s}}^{2}+\left\|\partial_{x} \theta(s)\right\|_{H^{s-1}}^{2}\right) d s \\
& +\int_{0}^{t}(1+s)^{-1 / 2}\left(\left\|\partial_{x} U(s)\right\|_{H^{s-2}}^{2}+\|v(s)\|_{H^{s-1}}^{2}\right) d s+C \int_{0}^{t}(1+s)^{-3 / 2}\|U(s)\|_{H^{s}}^{2} d s \\
& \leq C\left\|U_{0}\right\|_{H^{s}}^{2}+C \int_{0}^{t}(1+s)^{-1 / 2}\left\|\partial_{x} U(s)\right\|_{L^{\infty}}\left\|\partial_{x} U(s)\right\|_{H^{s-1}}^{2} d s \\
& +C \lambda \int_{0}^{t}(1+s)^{-1 / 2}\|z\|_{L^{\infty}}\left(\left\|\partial_{x} z(s)\right\|_{H^{s-2}}^{2}+\|v(s)\|_{H^{s-1}}\left\|\partial_{x} z(s)\right\|_{H^{s-1}}\right) d s .
\end{aligned}
$$

The last two terms in the right-hand side of (4.38) can be estimated as

$$
\begin{aligned}
\int_{0}^{t}(1+s)^{-1 / 2}\left\|\partial_{x} U(s)\right\|_{L^{\infty}}\left\|\partial_{x} U(s)\right\|_{H^{s-1}}^{2} d s & \leq M_{1}(t) \int_{0}^{t}(1+s)^{-3 / 2}\left\|\partial_{x} U(s)\right\|_{H^{s-1}}^{2} d s \\
& \leq M_{1}(t) D^{2}(t)
\end{aligned}
$$

Furthermore, exploiting the estimate $\|z(t)\|_{L^{\infty}} \leq C(1+t)^{-1 / 2} M_{0}(t)$, we get (cp. the estimate (4.34) in [13])

$$
\begin{aligned}
& \int_{0}^{t}(1+s)^{-1 / 2}\|z\|_{L^{\infty}}\left(\left\|\partial_{x} z(s)\right\|_{H^{s-2}}^{2}+\|v(s)\|_{H^{s-1}}\left\|\partial_{x} z(s)\right\|_{H^{s-1}}\right) d s \\
\leq & C M_{0}(t) \int_{0}^{t}(1+s)^{-1}\left(\left\|\partial_{x} z(s)\right\|_{H^{s-2}}^{2}+\|v(s)\|_{H^{s-1}}\left\|\partial_{x} z(s)\right\|_{H^{s-1}}\right) d s \\
\leq & C M_{0}(t) D^{2}(t) .
\end{aligned}
$$

Thus, plugging the estimates (4.39) and (4.40) into (4.38), then (3.20) holds true for $j=0$. It is sufficient to use induction on $j$ to show that (3.20) is fulfilled. Assume that (3.20) is 
satisfied for $j-1$; we will show that (3.20) is also valid for $j$. Indeed, taking $\mu=j-1 / 2$ in (4.9), and taking the summation over $k$ such that $j \leq k \leq s-j$, with $j \leq[s / 2]$, we get

$$
\begin{aligned}
& (1+t)^{j-1 / 2}\left\|\partial_{x}^{j} U(t)\right\|_{H^{s-2 j}}^{2}+\alpha \int_{0}^{t}(1+s)^{j-1 / 2}\left\|\partial_{x}^{j} y(s)\right\|_{H^{s-2 j}}^{2} d s \\
& +\frac{\delta}{\tau_{0}} \int_{0}^{t}(1+s)^{j-1 / 2}\left\|\partial_{x}^{j} w(s)\right\|_{H^{s-2 j}}^{2} d s \\
& \leq C\left\|\partial_{x}^{j} U_{0}\right\|_{H^{s-2 j}}^{2}+C \int_{0}^{t}(1+s)^{j-3 / 2}\left\|\partial_{x}^{j} U(s)\right\|_{H^{s-2 j}}^{2} d s \\
& +C \int_{0}^{t}(1+s)^{j-1 / 2}\left\|\partial_{x} U(s)\right\|_{L^{\infty}}\left\|\partial_{x}^{j} U(s)\right\|_{H^{s-2 j}}^{2} d s .
\end{aligned}
$$

Similarly, letting $\mu=j-1 / 2$ in (4.35), and taking the summation over $k$ so that $j \leq k \leq$ $s-j-2$ with $j \leq[s / 2]-1$, we find

$$
\begin{aligned}
& \int_{0}^{t}(1+s)^{j-1 / 2}\left(\left\|\partial_{x}^{j} \theta_{x}(s)\right\|_{H^{s-2 j-1}}^{2}+\left\|\partial_{x}^{j+1} U(s)\right\|_{H^{s-2 j-2}}^{2}+\left\|\partial_{x}^{j} v(s)\right\|_{H^{s-2 j-1}}^{2}\right) d s \\
\leq & C\left\|\partial_{x}^{j} U_{0}\right\|_{H^{s-2 j}}^{2}+C(1+t)^{j-1 / 2}\left\|\partial_{x}^{j} U(t)\right\|_{H^{s-2 j}}^{2} \\
& +C \int_{0}^{t}(1+s)^{j-3 / 2}\left\|\partial_{x}^{j} U(s)\right\|_{H^{s-2 j}}^{2} d s \\
& +C \int_{0}^{t}(1+s)^{j-1 / 2}\left(\left\|\partial_{x}^{j} y(s)\right\|_{H^{s-2 j}}^{2}+\left\|\partial_{x}^{j} w(s)\right\|_{H^{s-2 j}}^{2}\right) d s \\
& +C \int_{0}^{t}(1+s)^{j-1 / 2}\|z\|_{L^{\infty}}\left\{\left\|\partial_{x}^{j+1} z(s)\right\|_{H^{s-2 j-2}}^{2}\right. \\
& +\left\|\partial_{x}^{j} v(s)\right\|_{H^{s-2 j-2}}\left\|\partial_{x}^{j+1} z(s)\right\|_{H^{s-2 j-2}} \\
& \left.+\left\|\partial_{x}^{j+1} v(s)\right\|_{H^{s-2 j-2}}\left\|\partial_{x}^{j+2} z(s)\right\|_{H^{s-2 j-2}}\right\} d s .
\end{aligned}
$$

As above, and for $1 \leq j \leq[s / 2]-1$, then (4.41) $+\hat{\lambda}(4.42)$ gives for $\hat{\lambda}$ sufficiently small,

$$
\begin{aligned}
& (1+t)^{j-1 / 2}\left\|\partial_{x}^{j} U(t)\right\|_{H^{s-2 j}}^{2}+C \int_{0}^{t}(1+s)^{j-1 / 2}\left(\left\|\partial_{x}^{j} y(s)\right\|_{H^{s-2 j}}^{2}+\left\|\partial_{x}^{j} w(s)\right\|_{H^{s-2 j}}^{2}\right) d s \\
& +C \int_{0}^{t}(1+s)^{j-1 / 2}\left(\left\|\partial_{x}^{j} \theta_{x}(s)\right\|_{H^{s-2 j-1}}^{2}+\left\|\partial_{x}^{j+1} U(s)\right\|_{H^{s-2 j-2}}^{2}+\left\|\partial_{x}^{j} v(s)\right\|_{H^{s-2 j-1}}^{2}\right) d s \\
& \leq C\left\|\partial_{x}^{j} U_{0}\right\|_{H^{s-2 j}}^{2}+C \int_{0}^{t}(1+s)^{j-3 / 2}\left\|\partial_{x}^{j} U(s)\right\|_{H^{s-2 j}}^{2} d s \\
& +C \int_{0}^{t}(1+s)^{j-1 / 2}\left\|\partial_{x} U(s)\right\|_{L^{\infty}}\left\|\partial_{x}^{j} U(s)\right\|_{H^{s-2 j}}^{2} d s \\
& +C \int_{0}^{t}(1+s)^{j-1 / 2}\|z\|_{L^{\infty}}\left\{\left\|\partial_{x}^{j+1} z(s)\right\|_{H^{s-2 j-2}}^{2}+\left\|\partial_{x}^{j} v(s)\right\|_{H^{s-2 j-2}}\left\|\partial_{x}^{j+1} z(s)\right\|_{H^{s-2 j-2}}\right. \\
& \left.+\left\|\partial_{x}^{j+1} v(s)\right\|_{H^{s-2 j-2}}\left\|\partial_{x}^{j+2} z(s)\right\|_{H^{s-2 j-2}}\right\} d s
\end{aligned}
$$


where $C$ is depending on $\hat{\lambda}$. Since we assume that (3.20) holds for $j-1$ (hence (4.43) holds for $j-1$ instead of $j$ ), the second term in the right-hand side of (4.43) can be estimated as follows:

$$
\int_{0}^{t}(1+s)^{j-3 / 2}\left\|\partial_{x}^{j} U(s)\right\|_{H^{s-2 j}}^{2} d s \leq C\left\|U_{0}\right\|_{H^{s}}^{2}+C\left(M_{0}(t)+M_{1}(t)\right) D^{2}(t) .
$$

Since (4.41) holds also for $j=[s / 2]$, then from (4.38), (4.41), (4.43) and in order to show (3.20), it is suffices to prove the following two estimates:

$$
\int_{0}^{t}(1+s)^{j-1 / 2}\left\|\partial_{x} U(s)\right\|_{L^{\infty}}\left\|\partial_{x}^{j} U(s)\right\|_{H^{s-2 j}}^{2} d s \leq C M_{1}(t) D(t)^{2}, \quad \text { for } 1 \leq j \leq[s / 2],
$$

and

$$
\begin{aligned}
& \int_{0}^{t}(1+s)^{j-1 / 2}\|z\|_{L^{\infty}}\left\{\left\|\partial_{x}^{j+1} z(s)\right\|_{H^{s-2 j-2}}^{2}+\left\|\partial_{x}^{j} v(s)\right\|_{H^{s-2 j-2}}\left\|\partial_{x}^{j+1} z(s)\right\|_{H^{s-2 j-2}}\right. \\
& \left.+\left\|\partial_{x}^{j+1} v(s)\right\|_{H^{s-2 j-2}}\left\|\partial_{x}^{j+2} z(s)\right\|_{H^{s-2 j-2}}\right\} d s \leq C M_{0}(t) D(t)^{2}, \quad \text { for } 1 \leq j \leq[s / 2]-1 .
\end{aligned}
$$

The estimate (4.44) is obvious. Let us show (4.45). Indeed, we have

$$
\begin{aligned}
\int_{0}^{t}(1+s)^{j-1 / 2}\|z\|_{L^{\infty}}\left\|\partial_{x}^{j+1} z(s)\right\|_{H^{s-2 j-2}}^{2} d s & \leq C M_{0}(t) \int_{0}^{t}(1+s)^{j-1}\left\|\partial_{x}^{j+1} z(s)\right\|_{H^{s-2 j-2}}^{2} d s \\
& \leq C M_{0}(t) \int_{0}^{t}(1+s)^{j-1}\left\|\partial_{x}^{j+1} U(s)\right\|_{H^{s-2 j-2}}^{2} d s \\
& \leq C M_{0}(t) D(t)^{2} .
\end{aligned}
$$

On the other hand and as in [13], we have for $1 \leq j \leq[s / 2]-1$,

$$
\begin{aligned}
& \int_{0}^{t}(1+s)^{j-1 / 2}\|z\|_{L^{\infty}}\left\{\left\|\partial_{x}^{j} v(s)\right\|_{H^{s-2 j-2}}\left\|\partial_{x}^{j+1} z(s)\right\|_{H^{s-2 j-2}}\right. \\
& \left.+\left\|\partial_{x}^{j+1} v(s)\right\|_{H^{s-2 j-2}}\left\|\partial_{x}^{j+2} z(s)\right\|_{H^{s-2 j-2}}\right\} d s \leq C M_{0}(t) D(t)^{2} .
\end{aligned}
$$

Thus, the proof of Lemma 3.9 is completed.

\subsection{Proof of Lemma 3.10}

In order to prove (3.21), it is suffices to show the estimate

$$
\left\|\partial_{x}^{k} U(s)\right\|_{L^{2}} \leq C E_{0}(1+t)^{-1 / 4-k / 2}+C\left(M(t)^{2}+M_{0}(t) E(t)\right)(1+t)^{-1 / 4-k / 2},
$$

for $0 \leq k \leq[s / 2]-2$. 
By virtue of the Duhamel principle, the solution of problem (3.1) can be written as an integral equation of the form

$$
U(t)=e^{t \Phi} U_{0}+\int_{0}^{t} e^{(t-\tau) \Phi} G(U)_{x}(\tau) d \tau,
$$

where

$$
\left(e^{t \Phi} \omega\right)(x):=\mathcal{F}^{-1}\left[e^{t \hat{\Phi}(i \xi)} \hat{\omega}(\xi)\right](x)
$$

with $\hat{\Phi}(i \xi):=-(i \xi A+L)$ and $G(U):=(0,0,0, g(z), 0,0)$.

The arguments used to prove the estimate (4.46) are very similar to ones employed by Ide and Kawashima [13], so many of the details will therefore be omitted.

Taking the $L^{2}$ norm of (4.47), we conclude

$$
\begin{aligned}
\left\|\partial_{x}^{k} U(t)\right\|_{2} & \leq\left\|\partial_{x}^{k} e^{t \Phi} U_{0}\right\|_{2}+\int_{0}^{t}\left\|\partial_{x}^{k+1} e^{(t-\tau) \Phi} G(U)\right\|_{2} d \tau \\
& =I_{1}+I_{2}
\end{aligned}
$$

Since $e^{t \Phi} U_{0}$ is the solution of the linear problem, then from (3.5), we get for $l=k+1$

$$
I_{1} \leq C E_{0}(1+t)^{-1 / 4-k / 2} .
$$

The estimate of $I_{2}$ is standard. Let us split it into two parts:

$$
\begin{aligned}
I_{2} & =\int_{0}^{t / 2}\left\|\partial_{x}^{k+1} e^{(t-\tau) \Phi} G(U(\tau))\right\|_{2} d \tau+\int_{t / 2}^{t}\left\|\partial_{x}^{k+1} e^{(t-\tau) \Phi} G(U(\tau))\right\|_{2} d \tau \\
& =J_{1}+J_{2}
\end{aligned}
$$

and applying (3.5), with $l=k+1$, we infer that

$$
\begin{aligned}
J_{1} \leq & C \int_{0}^{t / 2}(1+t-\tau)^{-3 / 4-k / 2}\|G(U(\tau))\|_{L^{1}} d \tau \\
& +C \int_{0}^{t / 2}(1+t-\tau)^{-1 / 2-k / 2}\left\|\partial_{x}^{k+1} G(U(\tau))\right\|_{L^{2}} d \tau \\
\leq & C \int_{0}^{t / 2}(1+t-\tau)^{-3 / 4-k / 2}\|U(\tau)\|_{L^{2}}^{2} d \tau \\
& +C \int_{0}^{t / 2}(1+t-\tau)^{-1 / 2-k / 2}\left\|\partial_{x}^{2 k+1} G(U(\tau))\right\|_{L^{2}} d \tau \\
\leq & C M(t)^{2} \int_{0}^{t / 2}(1+t-\tau)^{-3 / 4-k / 2}(1+\tau)^{-1 / 2} d \tau \\
& +C \int_{0}^{t / 2}(1+t-\tau)^{-1 / 2-k / 2}\left\|\partial_{x}^{2 k+1} G(U(\tau))\right\|_{L^{2}} d \tau
\end{aligned}
$$


The first term in the right-hand side of (4.50) can be estimated as

$$
C M(t)^{2} \int_{0}^{t / 2}(1+t-\tau)^{-3 / 4-k / 2}(1+\tau)^{-1 / 2} d \tau \leq C M(t)^{2}(1+t)^{-1 / 4-k / 2} .
$$

Also, following [13], we have

$$
\int_{0}^{t / 2}(1+t-\tau)^{-1 / 2-k / 2}\left\|\partial_{x}^{2 k+l} G(U(\tau))\right\|_{L^{2}} d \tau \leq C M_{0}(t) E(t)(1+t)^{-1 / 4-k / 2} .
$$

Thus, we get

$$
J_{1} \leq C M(t)^{2}(1+t)^{-1 / 4-k / 2}+C M_{0}(t) E(t)(1+t)^{-1 / 4-k / 2} .
$$

On the other hand,

$$
\begin{aligned}
J_{2} & =\int_{t / 2}^{t}\left\|\partial_{x} e^{(t-\tau) \Phi} \partial_{x}^{k} G(U(\tau))\right\|_{2} d \tau \\
& \leq C \int_{t / 2}^{t}(1+t-\tau)^{-3 / 4}\left\|\partial_{x}^{k} G(U(\tau))\right\|_{L^{1}} d \tau+C \int_{t / 2}^{t}(1+t-\tau)^{-1 / 2}\left\|\partial_{x}^{k+2} G(U(\tau))\right\|_{L^{2}} d \tau .
\end{aligned}
$$

This implies that for $k \leq[s / 2]-1$ we have (cp. [13])

$$
\int_{t / 2}^{t}(1+t-\tau)^{-3 / 4}\left\|\partial_{x}^{k} G(U(\tau))\right\|_{L^{1}} d \tau \leq C M(t)^{2}(1+t)^{-1 / 4-k / 2} .
$$

Furthermore, for $k \leq[s / 2]-2$, we have

$$
\begin{aligned}
& C \int_{t / 2}^{t}(1+t-\tau)^{-1 / 2}\left\|\partial_{x}^{k+2} G(U(\tau))\right\|_{L^{2}} d \tau \\
& \leq C M(t) E(t) \int_{t / 2}^{t}(1+t-\tau)^{-1 / 2}(1+\tau)^{-k / 2-5 / 4} \\
& \leq C M_{0}(t) E(t)(1+t)^{-k / 2-3 / 4}
\end{aligned}
$$

where we have used the fact that $\left\|\partial_{x}^{k+2} G(U(\tau))\right\|_{L^{2}} \leq C\|U\|_{L^{\infty}}\left\|\partial_{x}^{k+2} U\right\|_{L^{2}}$ and the inequality

$$
\left\|\partial_{x}^{k+2} U\right\|_{L^{2}} \leq\left\|\partial_{x}^{k+2} U\right\|_{H^{s-2 k-4}} \leq E(t)(1+t)^{-k / 2-3 / 4}
$$

which holds for $k \leq[s / 2]-2$. Consequently, we obtain from above that

$$
J_{2} \leq C M(t)^{2}(1+t)^{-1 / 4-k / 2}+C M_{0}(t) E(t)(1+t)^{-k / 2-3 / 4} .
$$

Plugging all the above estimates into (4.48), we get

$$
\left\|\partial_{x}^{k} U(t)\right\|_{2} \leq C E_{0}(1+t)^{-1 / 4-k / 2}+\left(C M(t)^{2}+C M_{0}(t) E(t)\right)(1+t)^{-1 / 4-k / 2},
$$

for $0 \leq k \leq[s / 2]-2$. The remaining part of the proof is standard. We omit it. Thus the proof of Lemma 4.2 is completed. 


\subsection{Proofs of Lemma 3.8 and of Theorem 3.3}

First, we prove Lemma 3.8. Using the following interpolation inequality,

$$
\|U\|_{L^{\infty}} \leq \sqrt{2}\|U\|_{L^{2}}^{1 / 2}\left\|\partial_{x} U\right\|_{L^{2}}^{1 / 2}
$$

we can easily see that

$$
M_{0}(t) \leq C M(t),
$$

provided that $[s / 2]-2 \geq 1$, which leads to $s \geq 6$. Similarly, applying inequality (4.51), with $U_{x}$ instead of $U$, we get for $[s / 2]-2 \geq 2$ (that is, for $s \geq 8$ )

$$
M_{1}(t) \leq C M(t) .
$$

Thus, for $s \geq 8$, and from (3.20) and (3.21), we get by exploiting the above two inequalities

$$
(E(t)+D(t)+M(t))^{2} \leq C E_{0}^{2}+C(E(t)+D(t)+M(t))^{3}
$$

for all $t \in[0, T]$. By standard arguments we conclude (cf. [31]), that for sufficiently small $E_{0}$, we have

$$
E(t)+D(t)+M(t) \leq \hat{C}, \quad \forall t \in[0, T] .
$$

Indeed, let $x=(E(t)+D(t)+M(t))^{2}$ and $h(x)=C\left(E_{0}^{2}+x^{3 / 2}\right)-x$. Therefore, (4.52) implies $h(0)=C E_{0}^{2}$ and $f(x) \geq 0$. On the other hand, we have

$$
h^{\prime}(x)=\frac{3}{2} C x^{1 / 2}-1 \leq-\frac{1}{2},
$$

for $x$ small enough, say $0 \leq x \leq 1 /\left(9 C^{2}\right)$. From the identity $f(x)=f(0)+\int_{0}^{t} f^{\prime}(x) d x$, we deduce that $f$ changes its sign in $\left[0,2 C E_{0}^{2}\right]$. Let $\hat{C}$ be the first zero of the function $h$, then from above we deduce that (4.53) holds. This proves Lemma 3.8.

Finally, we prove Theorem 3.3. Multiplying the third equation in (3.1) by $\sigma(z / a) / a$, we may rewrite (3.3) as a symmetric-hyperbolic system, for which standard existence theorems (cp. [31]) yield, for $U_{0} \in H^{s}$, a unique local solution

$$
U \in C\left([0, T], H^{s}(\mathbb{R}) \cap C^{1}\left([0, T], H^{s-1}(\mathbb{R})\right)\right.
$$

to (3.3), where $T=T\left(\left\|U_{0}\right\|_{H^{s}}\right)>0$ only depends on the $H^{s}$-norm of $U_{0}$. Lemma 3.8 now gives the desired a priori estimate for the $H^{s}$-norm $\|U(t)\|_{H^{s}}$ for $0 \leq t \leq T$, allowing the usual continuation argument for a local solution.

\section{Acknowledgment}

A part of this work was done during the second author's visit to the Department of Mathematics and Statistics at the University of Konstanz, German, and he would like to thank the members of the Department for their kindness and warm hospitality. 


\section{References}

[1] F. Alabau-Boussouira. Asymptotic behavior for Timoshenko beams subject to a single nonlinear feedback control. Nonlinear Differ. Equ. Appl., 14:643-669, 2007.

[2] F. Ammar-Khodja, A. Benabdallah, J. E. Muñoz Rivera, and R. Racke. Energy decay for Timoshenko systems of memory type. J. Differential Equations, 194(1):82-115, 2003.

[3] C. Cattaneo. Sulla conduzione del calore. Atti. Sem. Mat. Fis. Univ. Modena., 3(83):83$101,1948$.

[4] D. S. Chandrasekharaiah. Thermoelasticity with second sound: A Review. Appl. Mech. Rev., 39(3):355-376, 1986.

[5] D. S. Chandrasekharaiah. Hyperbolic thermoelasticity: a review of recent literature. Appl. Mech. Rev., 51:705-729, 1998.

[6] M. Chester. Second sound in solids. Phys. Rev., 131:2013-2015, 1963.

[7] C. M. Dafermos and L. Hsiao. Development of singularities in solutions of the equations of nonlinear thermoelasticity. Quart. Appl. Math, 44(3):463-474, 1986.

[8] H. D. Fernández Sare and R. Racke. On the stability of damped Timoshenko systems - Cattaneo versus Fourier law. Arch. Rational Mech. Anal., 194(1):221-251, 2009.

[9] A. Guesmia and S. A. Messaoudi. General energy decay estimates of timoshenko systems with frictional versus viscoelastic damping. Math. Meth. Appl. Sci., 32(16):2102-2122, 2009.

[10] T. Hosono and S. Kawashima. Decay property of regularity-loss type and application to some nonlinear hyperbolic-elliptic system. Math. Mod. Meth. Appl. Sci., 16:1839-1859, 2006.

[11] W. J. Hrusa and S. A. Messaoudi. On formation of singularities on one-dimensional nonlinear thermoelasticity. Arch. Rational Mech. Anal., 111:135-151, 1990.

[12] K. Ide, K. Haramoto, and S. Kawashima. Decay property of regularity-loss type for dissipative Timoshenko system. Math. Mod. Meth. Appl. Sci., 18(5):647-667, 2008.

[13] K. Ide and S. Kawashima. Decay property of regularity-loss type and nonlinear effects for dissipative Timoshenko system. Math. Mod. Meth. Appl. Sci., 18(7):1001-1025, 2008.

[14] R. Ikehata. Diffusion phenomenon for linear dissipative wave equations in an exterior domain. J. Differential Equations, 186(2):633-651, 2002.

[15] R. Ikehata. Diffusion phenomenon for linear dissipative wave equations in an exterior domain. J. Differential Equations, 186(2):633-651, 2002. 
[16] R. Ikehata. Decay estimates by moments and masses of initial data for linear damped wave equations. Int. J. Pure Appl. Math., 5(1):77-94, 2003.

[17] S. Jiang and R. Racke. Evolution equations in thermoelasticity, volume 112 of Chapman \& Hall/CRC Monographs and Surveys in Pure and Applied Mathematics. Chapman \& Hall/CRC, Boca Raton, FL, 2000.

[18] L. Joseph and D. D. Preziosi. Heat waves. Rev. Mod. Physics, 61:41-73, 1989.

[19] T. Kubo and S. Kawashima. Decay property of regularity-loss type and nonlinear effects for some hyperbolic-elliptic system. Kyushu J. Math., 63(1):139-159, 2009.

[20] A. Matsumura. On the asymptotic behavior of solutions of semi-linear wave equations. Publ. Res. Inst. Math. Sci., 12(1):169-189, 1976.

[21] S. A. Messaoudi and M. I. Mustafa. On the stabilization of the Timoshenko system by a weak nonlinear dissipation. Math. Meth. Appl. Sci., 32(4):454-469, 2009.

[22] S. A. Messaoudi, M. Pokojovy, and B. Said-Houari. Nonlinear damped Timoshenko systems with second sound - global existence and exponential stability. Math. Meth. Appl. Sci., 32(5):505-534, 2009.

[23] S. A. Messaoudi and B. Said-Houari. Energy decay in a Timoshenko-type system with history in thermoelasticity of second sound. Submitted.

[24] S. A. Messaoudi and B. Said-Houari. Blow up of solutions with positive energy in nonlinear thermoelasticity with second sound. J. Appl. Math., 3:201-211, 2004.

[25] S. A. Messaoudi and B. Said-Houari. Exponential stability in one-dimensional nonlinear thermoelasticity with second sound. Math. Methods Appl. Sci., 28(2):205-232, 2005.

[26] S. A. Messaoudi and B. Said-Houari. Uniform decay in a Timoshenko-type system with past history. J. Math. Anal. Appl., 360(2):459-475, 2009.

[27] J. E. Muñoz Rivera and R. Racke. Mildly dissipative nonlinear Timoshenko systems, global existence and exponential stability. J. Math. Anal. Appl., 276:248-276, 2002.

[28] J. E. Muñoz Rivera and R. Racke. Global stability for damped Timoshenko systems. Discrete Contin. Dyn. Syst., 9(6):1625-1639, 2003.

[29] J. E. Muñoz Rivera and R. Racke. Timoshenko systems with indefinite damping. J. Math. Anal. Appl., 341(2):1068-1083, 2008.

[30] R. Quintanilla and R. Racke. Qualitative aspects of solutions in resonators. Arch. Mech. (Arch. Mech. Stos.), 60(4):345-360, 2008. 
[31] R. Racke. Lectures on nonlinear evolution equations. Initial value problems. Aspects of mathematics, E19. Friedrich Vieweg and Sohn: Braunschweig, Wiesbaden, 1992.

[32] R. Racke. Thermoelasticity with second sound-exponential stability in linear and non-linear 1-d. Math. Methods. Appl. Sci., 25(5):409-441, 2002.

[33] R. Racke. Asymptotic behaviour of solutions in linear 2-or 3-d thermoelasticity with second sound. Quart. Appl. Math, 61(2):315-328, 2003.

[34] R. Racke. Thermoelasticity. In Handbook of differential equations: evolutionary equations. Vol. V, Handb. Differ. Equ., pages 315-420. Elsevier/North-Holland, Amsterdam, 2009 .

[35] R. Racke and B. Said-Houari. Decay rates and global existence for semilinear dissipative Timoshenko systems. Quart. Appl. Math., to appear.

[36] R. Racke and Y. Wang. Nonlinear well-posedness and rates of decay in thermoelasticity with second sound. J. Hyperbolic Differ. Equ, 5(1):25-43, 2008.

[37] J. E. Muñoz Rivera and H. D. Fernández Sare. Stability of Timoshenko systems with past history. J. Math. Anal. Appl., 339(1):482-502, 2008.

[38] B. Said-Houari and A. Kasimov. Decay property of timoshenko system in thermoelasticity. Math. Meth. Appl. Sci, in press.

[39] I. E. Segal. Dispersion for non-linear relativistic equations, ii. Ann. Sci. Ecole Norm. Sup., 1(4):459-497, 1968.

[40] A. Soufyane and A. Wehbe. Exponential stability for the Timoshenko beam by a locally distributed damping. Electron. J. Differential Equations, 29:1-14, 2003.

[41] M. A. Tarabek. On the existence of smooth solutions in one-dimensional nonlinear thermoelasticity with second sound. Quart. Appl. Math., 50(4):727-742, 1992.

[42] H. Yang and A. Milani. On the diffusion phenomenon of quasilinear hyperbolic waves. Bulletin des Sciences Mathematiques, 124(5):415-433, 2000. 